\title{
Parallel image scanning with binary phase grating
}

\author{
K.-H. Brenner \\ brenner@ziti.uni-heidelberg.de
}

\section{R. Buschlinger}

\author{
Chair for Optoelectronics, University of Heidelberg, B6, 23-29, Bauteil C, 3.OG, 68131 Mannheim, \\ Germany \\ Chair for Optoelectronics, University of Heidelberg, B6, 23-29, Bauteil C, 3.OG, 68131 Mannheim, \\ Germany
}

We discuss a novel approach for parallel image scanning, whereby the intensity peaks are generated by a binary phase grating instead of a lens array. These gratings under certain conditions generate one light spot per grating period in several transverse planes. The diameter of the focal spot is not restricted by the numeric aperture according to Abbe's law and can be used to scan a specimen with many spots simultaneously. Thus, imaging speed could be increased significantly. We discuss the principle effect and approaches to scanning as well as challenges for a potential implementation. [DOI: 10.2971/jeos.2011.11024]

Keywords: diffraction grating, micro-optics, focussing, light concentration

\section{INTRODUCTION}

Microscopy applications like in system biology or quality inspection in industrial manufacturing generate an increasing demand for high-speed imaging techniques. An approach to satisfy this demand is spatial parallelization of the imaging system. In the case of wide-field imaging, the scanning time depends on the ratio of lens diameter to field of view of each individual imaging system. For image scanning, the required field of view is minimal, since the specimen is scanned pixel by pixel. To minimize the scan time, the number of lenses has to be maximized and therefore the size of the imaging systems has to be minimized. In the literature, Tiziani and Uhde [1] presented a confocal scanning microscope based on micro lenses with diameters of $125 \mu \mathrm{m}$. By decreasing the lens size, there is a practical limit at a lens diameter of approx. 8 wavelength, where the lens, due to diffraction, shows only limited amount of light concentration.

Recently, we observed (see Figure 1), that binary phase gratings in a certain configuration generate sharp spots for grating periods down to $3 \lambda$ [3]. Such gratings can be easily manufactured and enable a high degree of miniaturization.

\section{GRATING CONFIGURATIONS AND SPOT QUALITY}

In order to analyse, which grating configurations are practical for generating intensity patterns with distinct spots, the intensity on the optical axis was calculated for different $z$-positions and for a range of gratings with different fill factors using scalar diffraction theory. A high intensity in the resulting plot is an indicator for the existence of a spot located on the optical axis. With this method, spot generation was predicted for fill factors of around 0.7. The optimal fill factor for spot gen-

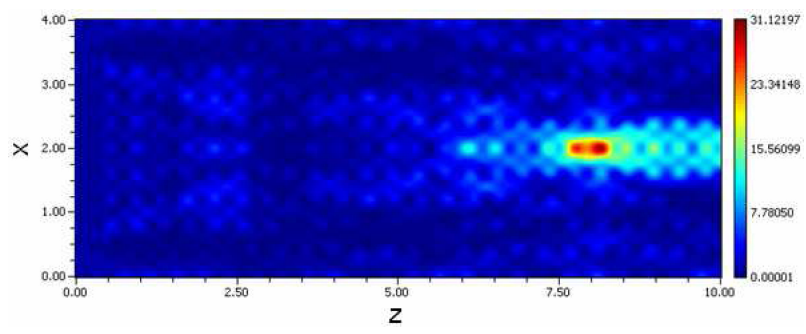

FIG. 1 Intensity in the x-z-plane behind a 2D-grating with $P_{x}=P_{y}=10 \lambda$, simulated with 2D-RCWA.

eration was found to depend only weakly on other grating parameters.

Another important parameter is the phase shift of the grating, which is controlled by changing the thickness of the grating layer. We expect maximal contrast for a phase shift of $\Delta \varphi=\pi$. For this configuration, we obtain an intensity pattern which is symmetric in $z$ around half of the grating's Talbot distance, $\frac{z_{T}}{2}$. There are two spots generated for each Talbot distance. For a phase shift of $\Delta \varphi=\frac{\pi}{2}$ or $\Delta \varphi=\frac{3 \pi}{2}$, we observe a similar plot only with slightly lower intensities on the optical axis. However, the intensity pattern is no longer symmetric around $\frac{z_{T}}{2}$. Instead, one of the spots in each Talbot length is missing. Thus, the number of z-planes with spot scan be decreased. In an imaging application, only one spot plane should contribute to the detected information in each scanning step. If there are several spot planes in the sample space, information might also be picked up from locations outside the intended object plane, leading to a superposition of the images for several values of $z$. The observed suppression of spots for special phase shift values can help to prevent this.

The third parameter of the grating configuration is the grat- 
ing period in relation to the wavelength, $\frac{P}{\lambda}$. The field in the $\mathrm{x}$-z-plane for several values for $\frac{P}{\lambda}$ was calculated with the rigorous coupled wave analysis (RCWA). We observed spot generation for values of $\frac{P}{\lambda}$ down to 3 . For ratios smaller than 6 , there are notable deviations from the results of scalar calculations. For a quantitative judgment of the spot quality for different grating periods, an effective numerical aperture was defined as

$$
N A_{e f f}=\frac{\lambda}{2 \cdot F W H M} .
$$

We observed an increase in effective numerical aperture, but a decrease in peak intensity with decreasing values of $\frac{P}{\lambda}$. For gratings with $\frac{P}{\lambda} \leq 4$, spots with an effective NA of 1 , i.e. with a FWHM of $\lambda / 2$ were obtained.

\section{IMAGING OF TRANSMITTED LIGHT}

If a specimen is placed inside one of the focal planes of a binary phase grating, its light transmission properties at the position of the spot can be measured in a detection plane such as a CCD behind the specimen.

The intensity values at any location behind the grating are formed by interference of light from neighboring grating periods. Thus, the specimen will affect not only the intensity pattern in the period where it is located, but also in neighboring periods. Therefore, we can expect a certain amount of crosstalk to neighboring pixels.

Figure 2 shows result from paraxial simulation, using an absorber with a diameter of $1 / 8$ of the detector pixel size. The period of the grating, $\mathrm{P}=10 \lambda \mathrm{s}$ matches the period of the detector array. The absorber is placed in plane of the focus and scanned laterally. For each absorber position, the detected intensity in the central detector pixel, $15 \mu \mathrm{m}$ behind the absorber is recorded.

Due to the thin element approximation, light in this calculation is only blocked in one discrete plane, leading to a comparatively small effect of the absorber. The result shows a distinct minimum with a FWHM close to the diameter of the absorber. The resolution for this case of $\frac{P}{\lambda}=10$ is approx. one wavelength. As mentioned before, a better resolution could be achieved for smaller periods, but this would also reduce the Talbot length and thus the effective working distance. When the absorber is shifted by an integer number of periods, also the crosstalk is visible, but the peak height is significantly smaller.

\section{IMAGING OF REFLECTED LIGHT}

Since a phase grating has no light absorption, it would also be possible to detect the light that is reflected back through the grating. In this case, the specimen is illuminated by the light focus and the reflected light passes the grating a second time. For this configuration, a beam splitter separating the incident and the reflected light is needed. Due to the geometry of a beamsplitter, the distance between the specimen and the detector must be at least the diameter of the beam splitter. In this case, the width of the grating and the maximal degree of

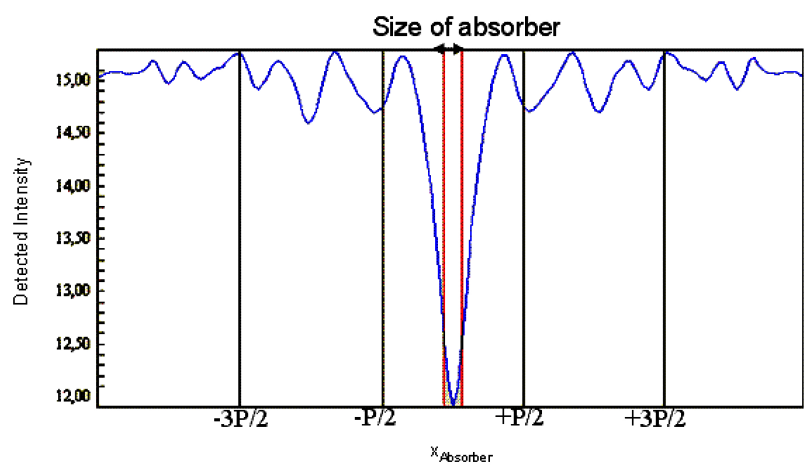

FIG. 2 Intensity behind a grating with $P=10 \lambda$ measured by a detector in the plane $z_{\text {det }}=z_{\text {spot }}+15 \mu \mathrm{m}$ for several placements of an absorbing pixel in the spot plane.

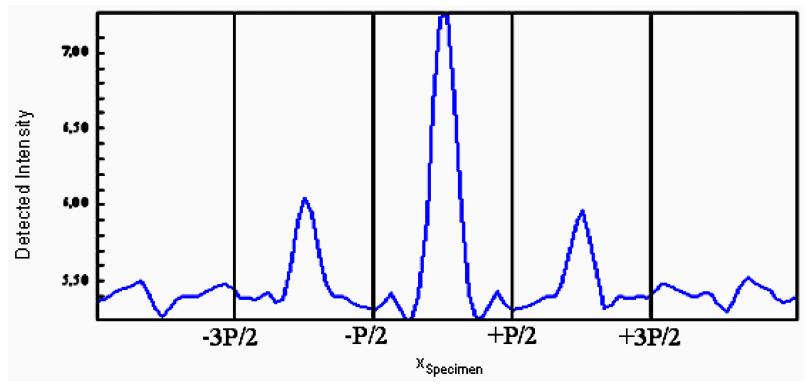

FIG. 3 Intensities measured by a detector in the plane $55 \mu \mathrm{m}$ before a grating with $P=10 \lambda$ for several placements of an absorbing pixel in the spot plane.

parallelization are limited by the maximal usable distance between object and detection plane. In our simulation, we assumed, that the intensity can be measured in a plane, located $55 \mu \mathrm{m}$ before the grating.

Figure 3 shows detected intensities in for several lateral positions of an absorbing pixel in the spot plane of a grating calculated with scalar diffraction theory. A sharp peak intensity is seen, when the absorber is moved into the spot of the detector's grating period. However, the side lobes are quite large at that distance.

\section{USE OF RELAY LENSES}

In order to avoid crosstalk due to diffraction, a relay-lens can be used for imaging the intensity pattern from the focal plane into the detection plane. The resolution of the overall system is mainly determined by the size of the focal spot and not by the relay lens. Thus, the relay lens only needs to be able to resolve roughly one grating period. The requirements on the relay lens in terms of geometric distortion are still relatively high, since the pitch of the phase grating has to match the pitch of the detector array. By using a combination of scanning spots generated by a phase grating and a relay lens, the pixel count of a detector can be increased significantly. If, for example, we use a one mega-pixel chip and we scan an array of $8 \times 8$ spots inside each pixel in parallel, a 64 mega-pixel detector would be obtained. In conventional microscopy there is always a tradeoff between resolution and field of view. Using massively parallel spot scanning, this tradeoff can be eliminated. 


\section{SCANNING}

In scanning microscopy, the focal spot has to be moved over the whole area that is to be imaged. In the present case, the scan has to occur only over the area of one grating period. One possibility to accomplish the scanning is mechanical movement of the grating. When the specimen is a liquid, mechanical scanning of the grating in close proximity could lead to disturbances, which would be problematic for image formation. In case of transmitted light imaging, one possible solution to this problem could be to use the self-imaging property of the Talbot effect. Thus, a spot in a larger distance from the grating could be selected for scanning. In reflected light imaging, this solution would be problematic, because an increase of the distance of the image plane from the grating would also increase the distance between the image and detection planes, which leads to crosstalk and therefore a lower signal to noise ratio.

A better option is to change the spot position by changing the angle of incidence of the illuminating plane wave. Simulations have shown, that the lateral spot position changes with the incident illumination angle similar to a lens. For a micro lens array, the change of the spot position $\Delta x$ is related to the angle of incidence $\vartheta$ by the relation $\Delta x=f \tan (\vartheta)$, where $f$ is the focal length of the lens. For a phase grating with $P=10 \lambda$ and a fill factor of 0.76 , we performed a simulation using scalar diffraction. The grating generates spots at $\mathrm{z}=8 \mu \mathrm{m}$. By changing the angle of incidence, we observe a spot shift $\Delta x$ as shown in Figure 4 (blue curve). For comparison, we also plotted the theoretical spot shift assuming a focal length of $8 \mu \mathrm{m}$. We see a small deviation, indicating that the effective focal length is smaller. With this technique, the spot can be scanned over the full period, but there is a noticeable decrease in spot quality for larger angles of incidence.

\section{SUMMARY}

We have analysed by numerical simulation, that phase gratings with a particular fill factor and height of the phase step generate sharp focus-like maxima of intensity. These spots have also been observed experimentally [3] before.

Utilizing these focal spots for parallel image scanning, a large

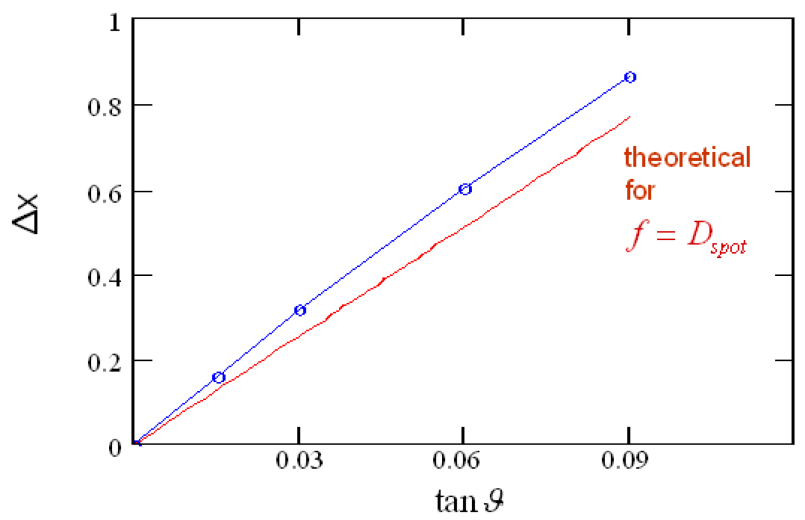

FIG. 4 Positions of a spot generated by a grating with and a fill factor of 0.76 for several angles of incidence. substrate can be scanned in the time that is required for scanning one single detector area. There are some constraints on the position of the detection plane. In both, reflected and transmitted light imaging, light has to be detected as close to the specimen as possible to ensure a correct mapping of intensity information to the detector pixel and to minimize crosstalk between the grating periods. If these constraints can not be met in a direct implementation, there is also the possibility of using a relay lens with unit magnification and low resolution, but low geometric distortion. Scanning over a single period can be accomplished either by mechanical movement of the grating or by changing the angle of incidence of the illuminating plane wave.

\section{References}

[1] H. J. Tiziani, H. M. Uhde, "Three dimensional analysis by a microlens array confocal arrangement" Appl. Opt. 33, 567-572 (1994).

[2] T. Tanaami, S. Otsuki, N. Tomosada, Y. Kosugi, M. Shimizu, H. Ishida, "High-speed 1 frame/ms scanning confocal microscope with a microlens and Nipkow disks" Appl. Opt. 41, 4704-4708 (2002).

[3] R. Buschlinger, K.-H. Brenner, "Light Focusing by binary phase gratings" in Proceedings to the fifth EOS Topical Meeting on Advanced Imaging Techniques (AIT) (EOS, Engelberg 2010). 